\title{
Towels versus hides: which are best at reducing acute stress in the newly hospitalised domestic cat (Felis sylvestris catus)?
}

\author{
by Arrandale, L. and Buckley, L.A.
}

Copyright, Publisher and Additional Information: This is the author accepted manuscript. The final published version (version of record) is available online via Taylor \& Francis

Please refer to any applicable terms of use of the publisher.

DOI: http://dx.doi.org/10.1080/17415349.2017.1343536

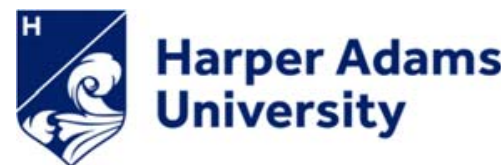

Arrandale, L. and Buckley, L.A. 2017. Towels versus hides: which are best at reducing acute stress in the newly hospitalised domestic cat (Felis sylvestris catus)? Veterinary Nursing Journal, 32(10), pp. 285-288. 


\section{Towels versus hides: which are best at reducing acute stress in the newly hospitalised domestic cat (Felis sylvestris catus)?}

Authors: Arrandale, L. and Buckley, L. A.

\section{Abstract}

The aim of this study was to identify whether towels over a cage or a box provided within a cage were better at reducing stress in the newly hospitalised cat. Forty - five cats were used, randomly allocated to one of three treatment groups: 1. Control, 2. Hide, 3. Screen. Temperature, heart rate (HR) and respiration rate (RR) were taken on admit and after 20 minutes. Cats were behaviourally scored for stress (CSS) on admit, and every minute for 20 minutes within their cage.

Control cats showed no reduction in HR or RR, but CSS did decline compared with the baseline $(\mathrm{P}=$ o.002). Compared to the Control, a significant decrease in HR and RR was observed for both Hide (HR, $\mathrm{P}=0.002 ; \mathrm{RR}, \mathrm{P}<0.001)$ and Screen cats $(\mathrm{HR}, \mathrm{P}=0.001 ; \mathrm{RR}, \mathrm{P}<0.001)$. HR decrease was similar between Hide and Screen cats but RR rate decrease was slightly more for Screen cats $(\mathrm{P}=0.049)$. CSS also declined for both Hide cats $(\mathrm{P}<0.001)$ and Screen cats $(\mathrm{P}<0.001)$, with Hide cats showing a tendency to reduce CSS more than Screen cats $(\mathrm{P}=0.054)$, and Screen cats a tendency to reduce CSS more than the Control condition $(\mathrm{P}=0.090)$. The CSS of Hide cats declined significantly more than Control cats $(\mathrm{P}=0.003)$

Differences between baseline CSS scores made interpretation difficult. Therefore, it is concluded that this study provided very limited, but positive, evidence that both enrichments may rapidly result in detectable reductions in feline stress levels. However, there is insufficient evidence to determine which enrichment method (if either) is better than the other.

\section{Introduction}

Hiding behaviours are a coping strategy used by cats exposed to stressors (Vinke et al., 2014). Providing a hide reduced stress levels in cattery - housed cats (Gourkow and Fraser, 2006; Kry and Casey, 2007; Vinke et al., 2014) and newly hospitalised cats (Buckley and Arrandale, 2017). However, it is not clear what aspect of providing a hide actually helps to reduce stress. For example, do hides reduce stress because the cat can remove itself from sight or because it is able to squeeze into a small space in which it feel more secure? Hides in which the cat can still be seen still reduce stress levels (Buckley and Arrandale, 2017). This suggests that actually being hidden from sight may not be necessary.

Despite the lack of research, covering kennels (e.g. with a towel) as an alternative method of reducing stress is utilised within veterinary practices and recommended by standard veterinary nursing textbooks (e.g. Orpet and Welsh, 2011). It is also more practical as reduced storage space is needed and they are easily laundered between cats. However, covering the cat kennel with a screen means that the cat cannot choose whether it wants to remain hidden or not. The ability to control exposure may be important to animal welfare (see: Wiepkema and Koolhaas, 1993). A screen may also not mimic the feature of the hide which makes hides effective at reducing stress in cats. Alternatively, it may be more effective as the cat may feel more secure hidden behind a screen. Therefore, the potential effects of screens on stress should be investigated further.

The study aimed to determine whether screens are as effective as hides at rapidly reducing the stress levels of newly hospitalised cats. It was hypothesised that both approaches would reduce stress compared with the conventional (no hide or screen) hospitalisation condition. It was expected that the screen would not be as effective as the hide due to the lack of control that the feline experiences under this condition.

3. Materials and methods

3.1. Subjects, facilities and treatment groups 
Forty - five cats (see table one) admitted for elective neutering at a charitable veterinary hospital were used. On arrival at the clinic, cats were randomly allocated (based on order of admittance) into one of three treatment groups:

1. Control group $(\mathrm{n}=15)$

2. Hide group $(\mathrm{n}=15)$

3. Screen group $(n=15)$

All cats were housed in a cat only ward, within a stainless steel cage $(65 \mathrm{~cm} \mathrm{x} 60 \mathrm{~cm} \times 70 \mathrm{~cm})$ with a metal barred door. The cage was lined with newspaper. The cattery cages were arranged in two tiers, with no visual access to any other caged cat. This was the control condition. The experiment groups also experienced this housing, with the addition of either a cardboard box $(40 \mathrm{~cm} \mathrm{x} 25 \mathrm{~cm} \mathrm{x} 25 \mathrm{~cm})$ within the cage (Hide group) or a blanket with a viewing hole $(10 \mathrm{~cm} \times 5 \mathrm{~cm})$ cut into it covering the door (Screen group).

\section{TABLE ONE GOES HERE}

\subsection{Experimental protocol}

Immediately on admission, the cat's temperature (digital thermometer, inserted until the thermometer beeped), respiration rate (RR) and heart rate (HR) (both 15 second recordings multiplied by 4), and baseline Cat - Stress - Score (CSS) were recorded. The cat was placed into a cage and the CSS recorded at minute intervals for 20 minutes using the behavioural approach reported by Kessler and Turner (1997). At the twenty minute point, the cat's temperature, respiration and HR were recorded again (as described above). These parameters were taken within the kennel to minimise confounding variables.

It was initially intended to video record the cats for later CSS assessment but pilot observations indicated that video quality was too poor for cats subjected to the screen condition.

\subsection{Statistical analysis}

All statistical tests were performed using Genstat (Version 15, VSN, Hemel Hempstead, UK). Due to the highly skewed nature of some of the data, non - parametric statistical tests were used to analyse the data and medians (plus inter-quartile range (IQR) were used to report the findings. The Kruskal Wallis ANOVA was used for the analysis of HR, respiration rate, temperature and CSS between the three groups.

Initial analyses were carried out on raw data for each time point observed. However, due to the small sample sizes, degree of variation between cats and the added complication of Hide cats showing a significantly higher stress level at o minutes compared to the Control and Screen cats it was necessary to control for this problem and to make the data more sensitive to intra-cat changes over time. Therefore, the data was converted to difference between two time points (e.g. CSS at o minutes minus CSS at 20 minutes) and the analyses re-run. Post-hoc analyses were carried out using the MannWhitney U test. The Wilcoxon matched pair test was used to analyse CSS, HR, RR and temperatures between start and end time periods of cats within all three groups.

$$
\text { 3.4. Ethics }
$$

The study was approved as per the Harper Adams Ethic committee protocol for undergraduate research projects. The primary experimental modifications applied (the hide or the screen) were expected to have positive welfare effects for the cats concerned, with only non - invasive or low invasive (temperature taking) data collection techniques employed.

4. Results

4.1. The effect of treatment group on CSS

Using the raw CSS data, a significant difference was observed between the groups at both o minutes $\left(\mathrm{H}_{2}\right.$ $=18.71, \mathrm{P}<0.001)$ and 20 minutes $\left(\mathrm{H}_{2}=8.797, \mathrm{P}=0.012\right)$. The median (I.Q.R.) CSS for the Control, Hide 
and Screen groups at o minutes was $3.8(2.5-4.3), 4.4(3.8-5)$ and $3.7(3.3-5.7)$ respectively. At 20 minutes, the median CSS for the Control, Hide and Screen groups was 3.3 (2.5 - 4.3), 3.6 (3-4.1) and 3.2 $(2.6-4.3)$ respectively. (See figure one).

\section{FIGURE ONE GOES HERE}

A post - hoc analysis indicated that the Hide group cats' CSS was significantly higher than the Control cats' CSS at both o minutes $\left(\mathrm{U}_{15,15}=20.5, \mathrm{P}<0.001\right)$ and 20 minutes $\left(\mathrm{U}_{15}, 15=61.5, \mathrm{P}=0.034\right)$. It was also significantly higher than the cats provided with a screen (o minutes: U15,15 = 25, P < 0.001; 20 minutes: U15,15 = 41.0, $\mathrm{P}=0.002$ ). A significant difference was not observed between the Control group and Screen group at either time point.

Using the converted data (the difference in CSS at each time point compared with the baseline, o minutes CSS), CSS significantly decreased over the 20 minutes for cats in all groups (Control cats: T12 = 3.o $\mathrm{P}=$ 0.002; Hide cats: $\mathrm{T} 15=0, \mathrm{P}<0.001$ : Screen cats: $\mathrm{T} 15=0, \mathrm{P}<0.001)$. Therefore, an inter - group analysis was carried out on the intra - cat differences between the CSS at o minutes and 20 minutes to identify whether a difference in the degree of stress reduction was similar between the groups. A significant difference between the groups in terms of how much the stress level of the cats had changed by 20 minutes $\left(\mathrm{H}_{2}=9.844, \mathrm{P}=0.007\right)$ was identified. The stress levels of the cats given a hide decreased significantly more than the Control cats $\left(\mathrm{U}_{15}, 15=43.5, \mathrm{P}=0.003\right)$. The relative decrease in the CSS of Screen cats was intermediary and showed only a tendency to differ from either the cats in the Control group $\left(\mathrm{U}_{15}, 15=71.5, \mathrm{P}=0.090\right)$ or Hide cats $\left(\mathrm{U}_{15}, 15=66.0, \mathrm{P}=0.054\right)$ (see figure two).

\section{FIGURE TWO GOES HERE}

\subsection{The effect of treatment on respiration rate}

The RR of the cats in each group did not differ significantly at the point of admission to the hospital ( $\mathrm{H}_{2}$ $=1.308, \mathrm{P}=0.518$ ). However, an analysis of the difference between $\mathrm{RR}$ at the point of admission and 20 minutes later indicated that the within cat change in RR over this period differed between the groups $\left(\mathrm{H}_{2}=18.71, \mathrm{P}<0.001\right.$; see table two for the post - hoc analysis). Compared to their baseline RR, Screen cats showed the greatest reduction (8 RPM), followed by Hide cats (4 RPM), with Control cats showing no decrease in RR. However, this difference was numerically very small and not detectable using an analysis of the end respiration rates of each group $\left(\mathrm{H}_{2}=1.042, \mathrm{P}=0.592\right)$.

\section{TABLE TWO GOES HERE.}

\subsection{The effect of treatment on heart rate}

The HR of the cats in each group did not differ significantly at the point of admission to the hospital $\left(\mathrm{H}_{2}=0.3345, \mathrm{P}=0.845\right)$ (see table three). However, an analysis of the difference between the HR at the point of admission and 20 minutes later indicated that the change in HR over this period also differed between the groups $\left(\mathrm{H}_{2}=12.54, \mathrm{P}=0.002\right.$, see table three for the post - hoc analysis $)$. Compared to their baseline HR, Screen cats showed the greatest reduction (6 BPM), followed by Hide cats (4 BPM), with Control cats showing no decrease in HR. However, this difference was numerically very small and not detectable using an analysis of the end heart rates of each group $\left(\mathrm{H}_{2}=1.781 ; \mathrm{P}=0.407\right)$.

\section{TABLE THREE GOES HERE.}

\subsection{The effect of treatment on temperature}

The temperature of the cats in each group did not differ significantly from each other either at the point of admission $\left(\mathrm{H}_{2}=0.2518, \mathrm{P}=0.878\right)$ or after 20 minutes $\left(\mathrm{H}_{2}=0.1474, \mathrm{P}=0.926\right)$. The median (I.Q.R.) temperature of the cats was as follows: o minutes: Control: 38.5 (38.3 - 38.6); Hide: 38.4 (38.3-38.6); Screen: 38.5 (38.4 - 38.5); 20 minutes: Control: 38.6 (38.3 - 38.6); Hide: 38.4 (38.3 - 38.7); Screen: 38.4 (38.4-38.5). Further, an intra-cat, within group analysis indicated that the temperatures did not 
change over this time period for any of the groups (Control: $\mathrm{T}_{7}=4.5, \mathrm{P}=0.125 ;$ Hide: $\mathrm{T}_{5}=4.5, \mathrm{P}=0.625$; Screen: $\left.\mathrm{T}_{5}=4.5, \mathrm{P}=0.625\right)$. Therefore, no further analysis was performed.

\section{Discussion}

This study provided very limited evidence that providing a hide or a screen could have a very small positive effect by causing a reduction in the levels of physiological and behavioural indicators of stress over the initial twenty minutes. However, the behavioural and physiological parameter measurements conflicted and it is not possible to draw firm conclusions about the relative benefits of providing a hide versus a screen on the stress levels of the cats, only that both methods had a positive effect on these levels.

This inability to be able to distinguish clearly between groups is probably partly due to the researcher not observing the cats for long enough. Operational issues (the cat ward was very narrow) and the busy nature of the PDSA (the researcher was continuously blocking the cat ward at a time when people traffic in the ward was very high) meant that the initial planned 30 minute observational period was reduced to twenty minutes. Perhaps with a 30 minute observation period the results obtained may have been clearer. There is a need to repeat this study over a longer time frame to better understand the potential relative efficacy of the methods. It seems unlikely that these small changes will have had any biological relevance within the time frame that the cats were observed. However, should the divergence in stress levels between the Control cats and either or both of the Hide or Screen cats increase, this could have very really welfare - enhancing benefits for how veterinary nurses manage the care of the feline immediately post - admit (and, often, shortly prior to anaesthesia). More research is required here and could be a useful study for the final year honours veterinary nursing student.

One interesting observation was how different both the baseline and 20 minutes post -admittance heart and respiration rates of the cats were compared to previous work by the authors (Buckley and Arrandale, 2017) with lower heart and respiration rates being reported for both Control and Hide cats at both admission and after 20 minutes. CSS was similar for both Control groups, with methodological issues making Hide CSS comparisons more difficult. This underscores the importance of baseline measurements when undertaking a study of this type in order to understand and interpret the findings. The behavioural and physiological differences observed between the two studies probably reflect the different nature of the two veterinary practices. The former study utilised a quiet small animal practice with few inpatients and low noise levels. Whereas, the current study took place in a very busy practice with a high caseload noise levels. Anecdotally, one veterinarian employed at a busy emergency clinic remarked that if a newly hospitalised cat had a HR in the 120 (as observed by Buckley and Arrandale, 2017) they might be concerned about possible health issues. This might reflect the risk that veterinary professionals are adapted to expect higher heart rates and not necessarily recognising that it might be a sign of poorer feline welfare. It also highlights the increased importance of considering measures to reduce feline stress when cats are admitted to busy clinics.

In conclusion, there is very limited evidence that both a screen and a hide have a positive effect on the parameters of stress measured but not sufficient evidence to allow the relative efficacy of each method to be determined. However, in the interim, there was no evidence that providing either a screen or a hide had negative effects over the period observed and may be beneficial so it may be prudent to consider providing one (or both) of them until proven either way.

\section{Acknowledgements}

The authors would like to thank the Universities Federation for Animal Welfare for providing an Animal Welfare Summer Scholarship to Laura Arrandale whilst a final year undergraduate student at Harper Adams University. The staff members at the People's Dispensary for Sick Animals Animal Hospital (Southampton) are also thanked for allowing this study to be carried out at their facility.

\section{References}


Buckley, L. A. and Arrandale, L. 2017. The use of hides to reduce acute stress in the newly hospitalised domestic cat (Felis sylvestris catus). Veterinary Nursing Journal IN PRESS, AWAITING

\section{CONFIRMATION OF ISSUE NUMBER.}

Gourkow, N. and Fraser, D. 2006. The effect of housing and handling practices on the welfare, behaviour and selection of domestic cats (Felis sylvestris catus) by adopters in an animal shelter. Animal Welfare 15: $371-377$

Kessler, M. R. and Turner, D.C. 1997. Stress and adaptation of cats (Felis sylvestris catus) housed singly, in pairs and in groups in boarding catteries. Animal Welfare 6(3): 243- 254

Kry, K. and Casey, R. 2007. The effect of hiding enrichment on stress levels and behaviour of domestic cats (Felis sylvestris catus) in a shelter setting and the implications for adoption potential. Animal Welfare 16(3): $375-383$

Vinke, C. M., Godin, L. M. and van der Leij, W. J. R. 2014. Will a hiding box provide stress reduction for shelter cats? Applied Animal Behaviour Science 160: 86 - 93

Wiepkema, P. R. and Koolhaas, J. M. 1993. Stress and animal welfare. Animal Welfare 2(3): 195 - 218 